\title{
The Research on Optimization of the Multiphase Flow Field of Biogas Plant by Using CFD Software
}

\author{
Ruyi Huang ${ }^{1,2}$, Yan Long ${ }^{1}$, Tao Luo ${ }^{1,2}$, Zili Mei ${ }^{1}$, Jun Wang ${ }^{2}$ and Enshen Long ${ }^{2}$ \\ 1. BIOMA (Biogas Institute of Ministry of Agriculture), Asian-Pacific Biogas Research \& Training Center, Chengdu 610041, China \\ 2. College of Architecture and Environment, Sichuan University, Chengdu 610065, China
}

Received: November 10, 2013 / Accepted: January 02, 2014 / Published: June 30, 2014.

\begin{abstract}
The inner flow field of a biogas plant can be optimized by agitating the feedstock to be evenly distributed for a rising biogas production rate. A hydraulic agitator can be installed in the digester with outlets far above the bottom. Hydraulic mixing is essential in a solid-liquid two-phase flow process, in which large solid particles can be found at the initial stage and turn to being high-concentration viscous liquid (non-Newtonian fluid). A $0.75 \mathrm{~m}^{3}$ digester was taken as a case study with CFD (computational fluid dynamics) software. The basic pattern was simulated by using water as the medium and the pattern of pseudo plastic fluid state was simulated by the Euler-Euler Model, then the effect of optimized design with bottom inflow and high dispersed outlets could be verified. Viewed from the mixing effects, the velocity of $0.6 \mathrm{~m} / \mathrm{s}$ is better than $1 \mathrm{~m} / \mathrm{s}$ for water medium, while $1 \mathrm{~m} / \mathrm{s}$ better than $0.6 \mathrm{~m} / \mathrm{s}$ for pseudo plastic fluid medium.
\end{abstract}

Key words: Biogas plant, mixing, multiphase flow, CFD, flow simulation.

\section{Introduction}

As the product of biogas digestion from straw, livestock manure, domestic sewage and other pollutants, $\mathrm{CH}_{4}$ (methane) is a potent greenhouse gas with strong greenhouse effect from direct emission in spite of being high-value energy. Therefore, biogas plant is a practical solution to the problems of rural energy shortage, environmental pollution and an effective way for carbon emission mitigation. According to the statistics of the Ministry of Agriculture of China, by the end of 2012, the number of household anaerobic digesters in China had reached 40,270,000 and annual gas production 13.08 billion cubic meters. Nevertheless, the current methods of static feeding, discharging and distribution of the feedstock in digesters, lacking manual intervention, result in low slurry liquidity and low biogas production rate [1]. Mixing of digesters is needed to break the

Corresponding author: Mei Zili, full professor, research field: biogas.E-mail: 13880233242@163.com. static fermentation and optimize the flow field [2]. About the three major mixing techniques involving mechanical, hydraulic and pneumatic [3-5]; It remains in dispute over whether technique is the best or how to assess the mixing effect [6]. CFD (computational fluid dynamics) software can be used to calculate and visualize the field change in the digesters caused by mixing (Vesvikar and Al-Dahhan) [7]; Karim [8] tested that the flow field matches the fact simulated using CFD by CARPT (computer automated radioactive particle tracking) and $\mathrm{CT}$ (computed tomography); Vesvikar [9] made further experimental verification on the flow field parameters such as velocity distribution calculated by CFD simulation. $\mathrm{Wu}$ [10] stated that the physical characteristics of bio-slurry is similar to that of the fluid whose flow field during mixing is a two phase process of feedstock and water, and simulation calculation was done on the two phase flow of the non-Newton fluid in the digester with CFD software. Shen, et al. [11] made comparative analysis on the single phase flow and two phase flow in 
the fermentation slurry; but dispute remains in the circle on which of the three mixing methods is superior. For instance, as found by $\mathrm{Wu}$ [12], flow field of mechanical stirring is the best, followed by pneumatic stirring, next to hydraulic stirring by CFD simulation. Michelan et al. [13] experimentally studied that the backflow mixing using biogas produced by the system is more effective than other mixing methods, though unstable, which might result from poor backflow mixing effect of low biogas yield from the system, as stated by Brito [14]. Lee [15] experimentally proved that the linear increases the pressure of pneumatic stirring, but the volumetric biogas producing rate remains around $1.25 \mathrm{~L} \cdot \mathrm{L}^{-1} \cdot \mathrm{d}^{-1}$, unable to continue to ascend, while Karim [16] stated by experiment that the digesters mixed by slurry recirculation, impeller, and biogas recirculation produced approximately $29 \%$, $22 \%$ and $15 \%$ more biogas than unmixed digester, respectively, which verified that the hydraulic mixing is the best method. In author's previous quantitative description on the effect of several models of hydraulic mixing with CFD software, the bottom inflow mixing was stated as the best in hydraulic mixing, and the high dispersed outlets [16] were optimized. However, the in-depth study of the inlet velocity of hydraulic mixing has not been reported. In this paper, a cylindrical anaerobic digester with $0.8 \mathrm{~m}$ diameter, $1.5 \mathrm{~m}$ height, and total volume of $0.75 \mathrm{~m}^{3}$ have been taken as a case study. The mixing effect of different inlet velocity is quantitatively studied, and the best inlet velocity of hydraulic mixing will be stated.

\section{Simulation Objects and Mathematical Model}

\subsection{Simulation Objects and Conditions}

To analyze the effect of hydraulic mixing on the internal flow field, a circulatory system is built with cyclic mixing run by a pump for inflow from the digester bottom and outflow through four dispersed outlets. The digester is $0.8 \mathrm{~m}$ in the bottom diameter, $1.5 \mathrm{~m}$ in height, the effective volume for the fermentation slurry is $0.60 \mathrm{~m}^{3}$, and the gas storage capacity $0.15 \mathrm{~m}^{3}$. The four outlet pipes are $1.1 \mathrm{~m}$ high from the bottom of the digester. As shown in Fig. 1.

\subsection{Mathematical Model}

Biogas slurry is a low concentration solution at the initial stage of biogas fermentation. Its flow liquidity
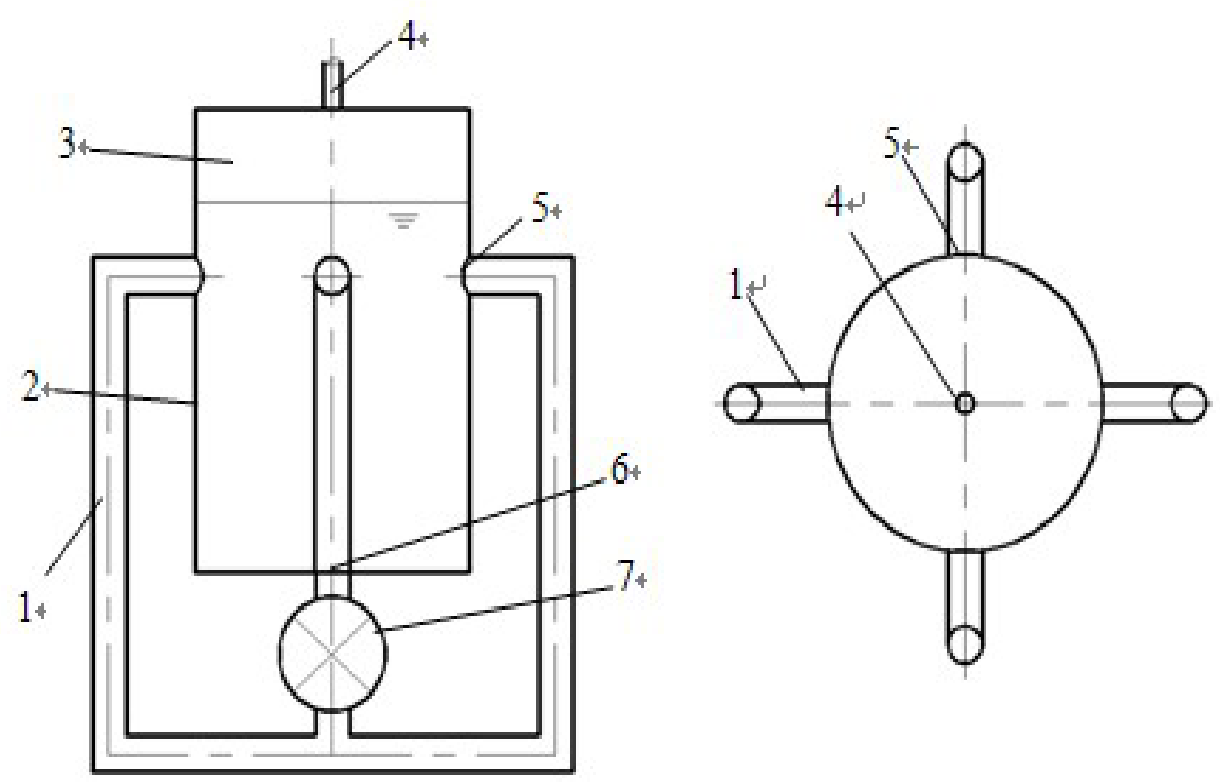

Fig. 1 Schematic diagram of digester with dispersed pressure outlets.

1: liquid pipe; 2: slurry chamber; 3: gas storage chamber; 4: gas pipe; 5: outlet; 6: inlet; 7: pump. 
is similar to that of water; therefore the liquid water is used as the medium to simulate the flow field. This can be used as a basic flow field of fluid in the anaerobic digester. In the later fermentation stage, straw was gradually converted into a state of pseudoplastic fluid with high concentration and viscosity [17]; the mixing at this stage caused a liquid-liquid two-phase flow of high-concentration straw solution and water. In this case, the Euler-Euler Model simulation is the preferred method for simulation calculation.

2.2.1 Mathematical Model Using Water as the Medium for Basic Flow Field

Not counting the time change, the fluid flow continuity equation is shown below:

$$
\frac{\partial\left(\rho u_{x}\right)}{\partial x}+\frac{\partial\left(\rho u_{y}\right)}{\partial y}+\frac{\partial\left(\rho u_{z}\right)}{\partial z}=0
$$

where, $\rho$ is fluid density.

The momentum equations are shown below:

$$
\begin{aligned}
& \nabla \cdot\left(\rho u_{x} \vec{u}\right)=-\frac{\partial \rho}{\partial x}+\frac{\partial \tau_{x x}}{\partial x}+\frac{\partial \tau_{x y}}{\partial y}+\frac{\partial \tau_{x z}}{\partial z}+\rho f_{x} \\
& \nabla \cdot\left(\rho u_{y} \vec{u}\right)=-\frac{\partial \rho}{\partial y}+\frac{\partial \tau_{x y}}{\partial x}+\frac{\partial \tau_{y y}}{\partial y}+\frac{\partial \tau_{z y}}{\partial z}+\rho f_{y} \\
& \nabla \cdot\left(\rho u_{z} \vec{u}\right)=-\frac{\partial \rho}{\partial z}+\frac{\partial \tau_{x z}}{\partial x}+\frac{\partial \tau_{y z}}{\partial y}+\frac{\partial \tau_{z z}}{\partial z}+\rho f_{z}
\end{aligned}
$$

where, $\tau$ is fluid shear stress, $u$ is velocity magnitude, and $\vec{u}$ is average velocity. And in the formula, $\nabla$ is the Hamilton differential operator:

$$
\nabla=i \frac{\partial}{\partial x}+j \frac{\partial}{\partial y}+k \frac{\partial}{\partial z}
$$

\subsubsection{Euler-Euler Model}

Assuming the two fluid phases are $p$ and $q$, the flow continuity equation of the $q$ phase is shown below:

$$
\frac{\partial}{\partial t}\left(\alpha_{q} \rho_{q}\right)+\nabla \cdot\left(\alpha_{q} \rho_{q} v_{q}\right)=\sum_{q=1}^{n} \dot{m}_{p q}
$$

where, $t$ is time, $\alpha_{q}$ is volume friction of phase $q$, $\rho_{q}$ is density of phase $q, v_{q}$ is velocity of phase $q$, $\dot{m}_{p q}$ is mass transfer from phase $p$ to phase $q$, and $\dot{m}_{q p}$ is the mass transfer from phase $q$ to phase $p$.

The momentum conservation equation of the $q$ phase is as the follows:

$$
\begin{gathered}
\nabla \cdot\left(\alpha_{q} \rho_{q} \vec{v}_{q} \vec{v}_{q}\right)=-\alpha_{q} \nabla p+\nabla \cdot \overline{\tau_{q}}+\sum_{p=1}^{n}\left(\vec{R}_{p q}+\dot{m}_{p q} \vec{v}_{p q}\right) \\
+\alpha_{q} \rho_{q}\left(\vec{F}_{q}+\vec{F}_{l i f, q}+\vec{F}_{v m, q}\right)
\end{gathered}
$$

In the formula, $\overline{\overline{\tau_{q}}}$ is the stress-strain tensor of the $q$ phase:

$$
\overline{\overline{\tau_{q}}}=\alpha_{q} \mu_{q}\left(\nabla \vec{v}_{q}+\nabla \vec{v}_{q}^{T}\right)+\alpha_{q}\left(\lambda_{q}-\frac{2}{3} \mu_{q}\right) \nabla \cdot \vec{v}_{q} \overline{\bar{I}}
$$

where, $\mu_{q}$ and $\lambda_{q}$ is shear viscosity and volume viscosity, $\vec{F}_{q}$ is external volume force, $\vec{F}_{l i f t, q}$ is lift force, $\vec{F}_{v m, q}$ is virtue mass force, $\vec{R}_{p q}$ is interphase interaction force, $\vec{v}_{p q}$ is interphase velocity, and $\overline{\bar{I}}$ is unit strain tensor.

The continuity equation of the volume fraction of the $q$ phase is shown below:

$$
\nabla \cdot\left(\alpha_{q} \vec{v}_{q}\right)=\frac{1}{\rho_{q}} \sum_{p=1}^{n} \dot{m}_{p q}
$$

\subsection{Numerical Methods}

As the biogas digester is mainly composed of the slurry chamber and the gas storage chamber, hydraulic mixing affects mainly the slurry area, the liquid column is taken as the calculation basis. The digester model is simplified into a $1.2 \mathrm{~m}$-high liquid column with a circular hole of $0.05 \mathrm{~m}$ diameter at the bottom, and four circular holes each with a diameter of $0.1 \mathrm{~m}$ are evenly dispersed at the position of $1.1 \mathrm{~m}$-high in the liquid column. Using control volume method, totally $3,334,851$ grid elements are generated, and the grid model is shown in Fig. 2.

\section{Comparison of Simulation Results in Different Conditions}

Simulation of varied media and varied flow rate helps develop the flow field forms under different operation conditions, which indicates the impact of inlet flow velocity on varied media.

\subsection{Simulation Using Water as the Medium}

First, water is used as the medium, and the simulation is done by changing inlet flow velocity. 


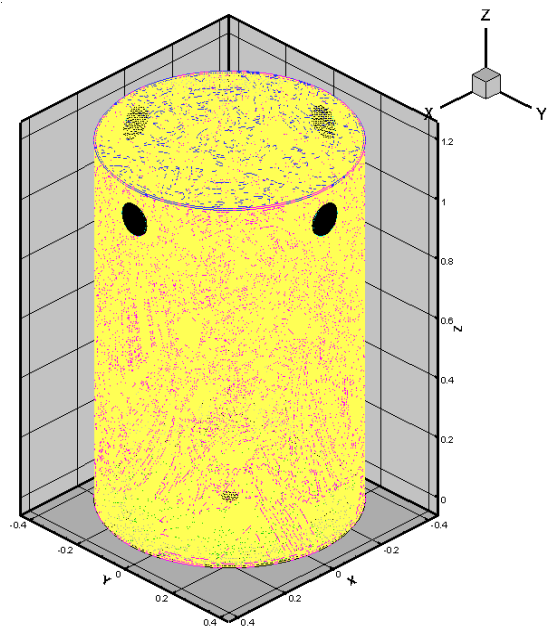

Fig. 2 The grid of liquid column.

\section{1 .1 Inlet Velocity of $0.3 \mathrm{~m} / \mathrm{s}$}

Fig. 3 is a simulation result from the inlet velocity of $0.3 \mathrm{~m} / \mathrm{s}$. From the figure, the velocity vectors distribution is relatively even, and the majority of kinetic energy is found in high area. At the initial stage of fermentation, as most feedstock float in the high area, the kinetic energy concentrating in the high area can help to mix up the slurry out of gravity and enhance the fermentation efficiency [16]. However, as the total kinetic energy is not abundant in this condition, a higher inlet velocity is considered.

\subsubsection{Inlet Velocity of $0.6 \mathrm{~m} / \mathrm{s}$}

Fig. 4 is a simulation result from the inlet velocity of $0.6 \mathrm{~m} / \mathrm{s}$. From the figure, the velocity vectors distribution remains relatively even, which gives little impact on the kinetic energy distribution in low area, but as the total kinetic energy is much more abundant, the liquidity of flow field is greatly enhanced.

\subsubsection{Inlet Velocity of $1 \mathrm{~m} / \mathrm{s}$}

Fig. 5 is a simulation result when the inlet velocity is raised to $1 \mathrm{~m} / \mathrm{s}$. The figure shows that the kinetic energy is more abundant than in the last working condition, but the distribution is obviously less even. Especially in the $x=0$ section, in the area at a height of $z=0.5 \mathrm{~m}$ and $z=0.9 \mathrm{~m}$, stratification is very obvious, which shows that the mixing from high-energy consumption is not helpful for the even mixture of slurry instead.

\subsubsection{Inlet Velocity of $1.5 \mathrm{~m} / \mathrm{s}$}

Fig. 6 is a simulation result when the inlet velocity grows to $1.5 \mathrm{~m} / \mathrm{s}$. From the figure, it is shown that despite the abundant total kinetic energy, the stratification is more obvious. Thus, when the inlet velocity reaches a certain rate, the flow pattern would be worsened with the velocity rise.

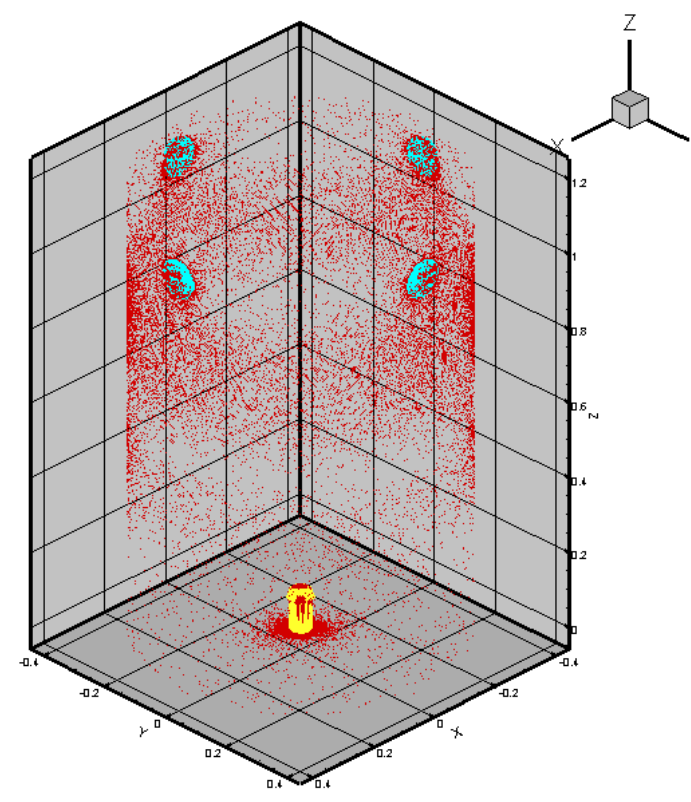

(a) Velocity vector diagram of $3 \mathrm{D}$

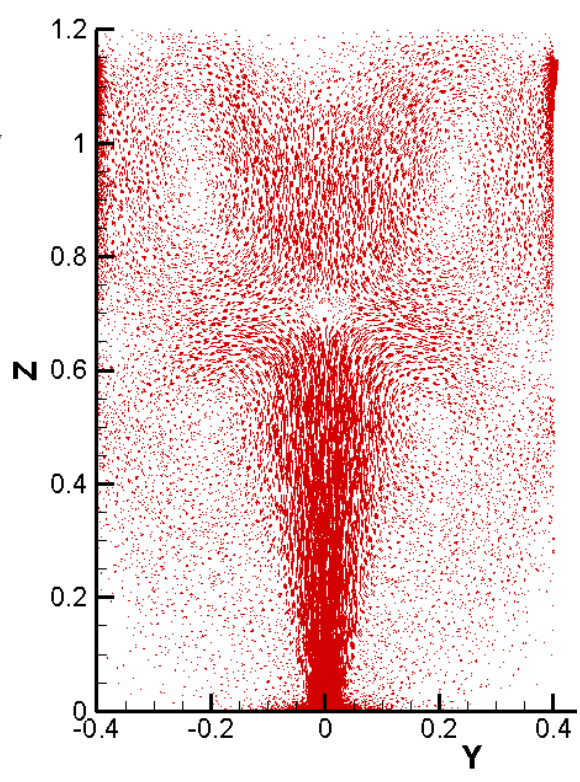

(b) Velocity vector diagram of $x=0$ section

Fig. 3 Inlet velocity $=0.3 \mathrm{~m} / \mathrm{s}$, velocity vector diagrams of $3 D$ and $x=0$ section. 


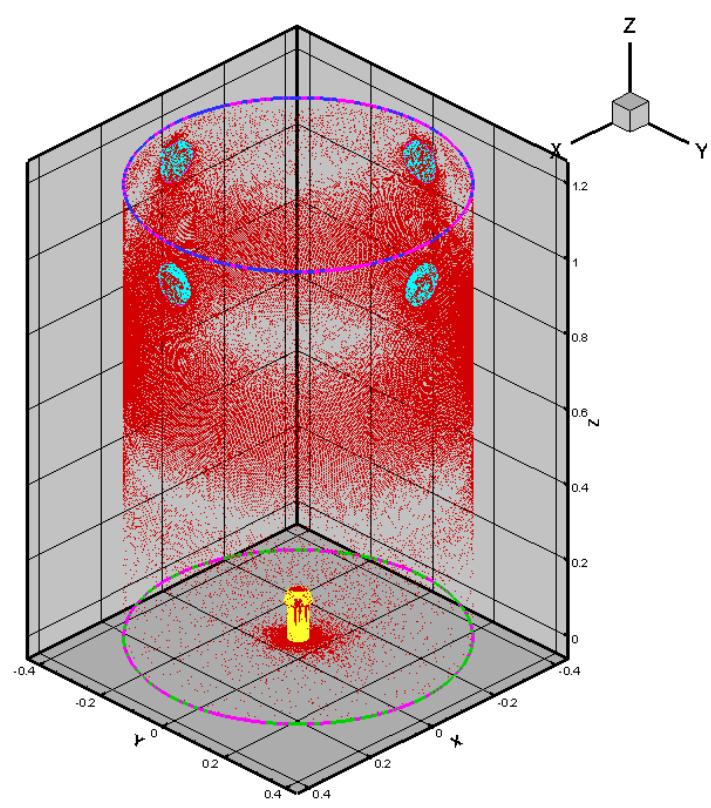

(a) $3 \mathrm{D}$

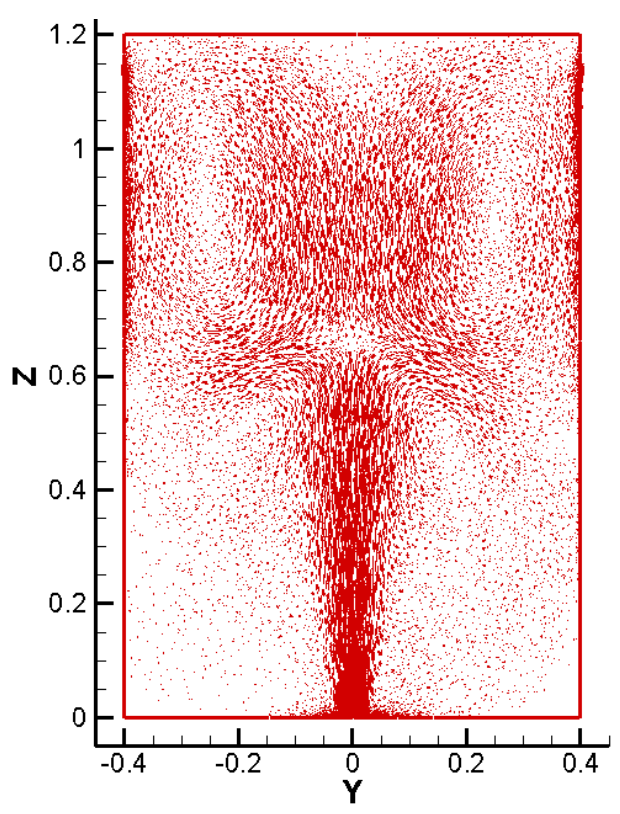

(b) $x=0$ section

Fig. 4 Inlet velocity $=0.6 \mathrm{~m} / \mathrm{s}$, velocity vector diagrams.

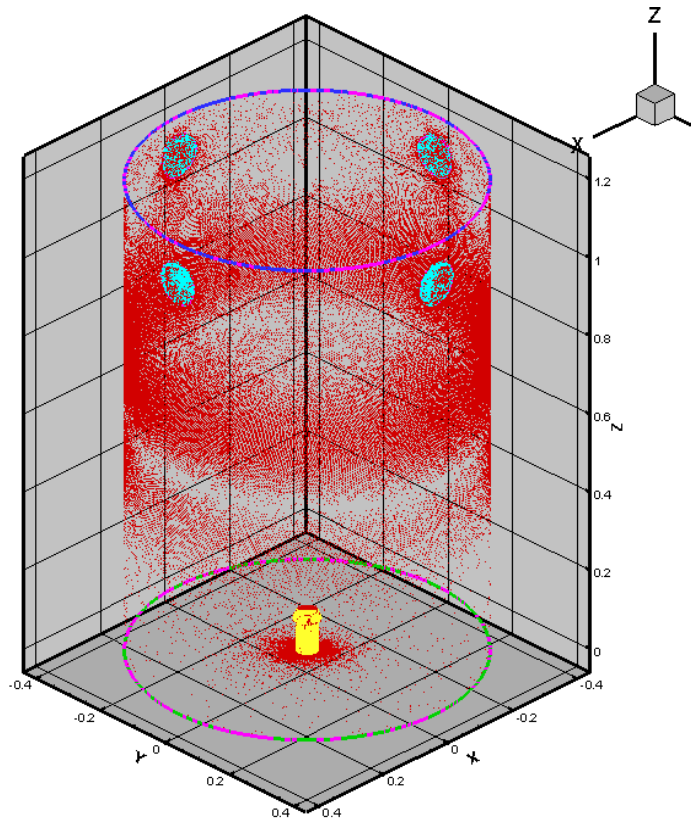

(a) $3 \mathrm{D}$

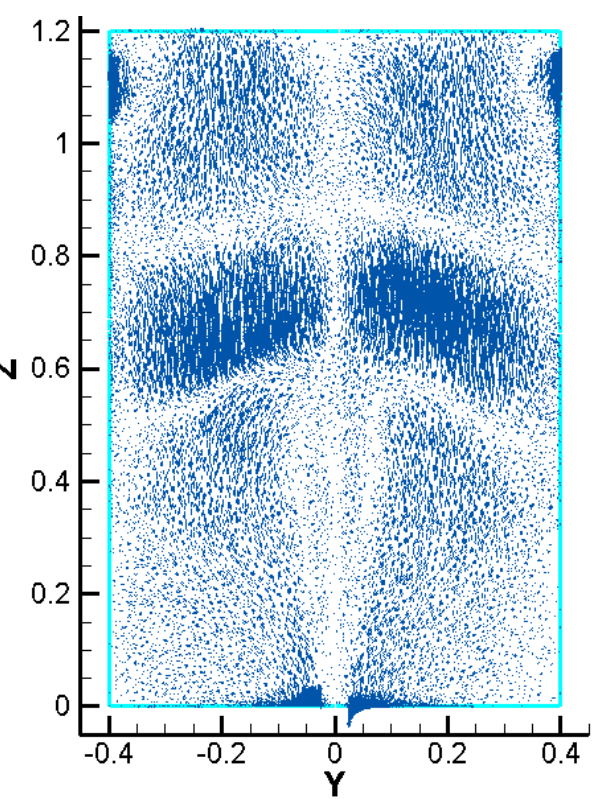

(b) $x=0$ section

Fig. 5 Inlet velocity $=1 \mathrm{~m} / \mathrm{s}$, velocity vector diagrams.

3.2 Simulation of Liquid-Liquid Two-Phase Flow in the Later Stage of Fermentation

Fig. 7 shows the morphological changes of straw after 30 days fermentation. At the initial stage of fermentation, the straw is mainly made up of complete particles or strips when it is fed into the digester at the beginning, and it is isolated from water relatively.
However, after continuous fermentation, the straw gradually becomes pseudo plastic fluid [18].

At the later stage, the flow pattern in slurry is greatly different from that of pure water. The straw fluid looks like a viscous fluid, and the flow field is a liquid-liquid two-phase flow of water and straw. Uniformity about the mixture can be illustrated by the volume fraction 


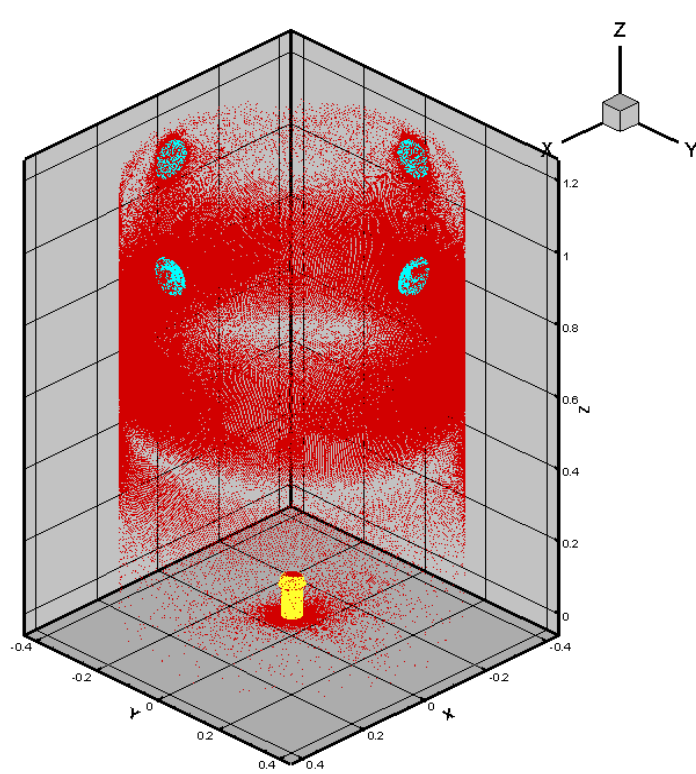

(a) $3 \mathrm{D}$

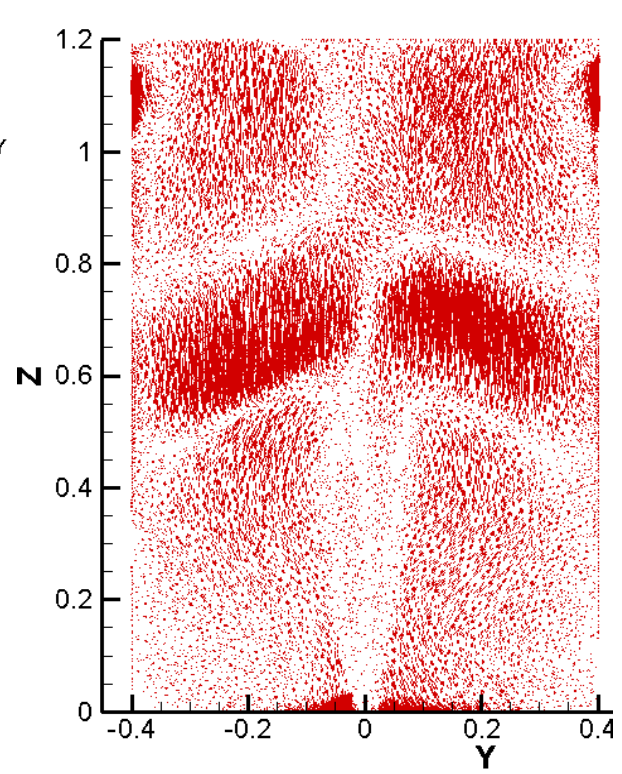

(b) $x=0$ section

Fig. 6 Inlet velocity $=1.5 \mathrm{~m} / \mathrm{s}$, velocity vector diagrams.
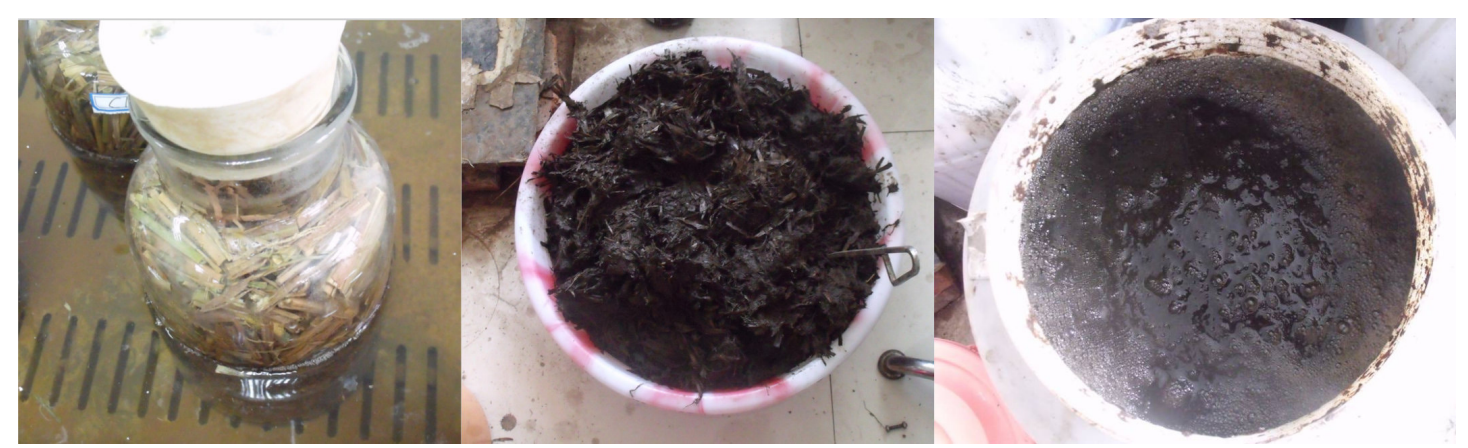

Fig. 7 The morphological changes of straw during continuous fermentation.

distribution of the secondary phase (straw) [9] in the flow field.

According to the experiment, after 30 days of full fermentation, the TS (total solid) concentration is $12.45 \%$, density $967.56 \mathrm{~kg} / \mathrm{m}^{3}$, kinematic viscosity coefficient $0.013 \mathrm{~m}^{2} / \mathrm{s}$. Simulation conditions are determined by the above physical parameters.

\section{2 .1 Inlet Velocity of $0.6 \mathrm{~m} / \mathrm{s}$}

Fig. 8 shows the volume fraction contours in the secondary phase (straw) with the inlet velocity of 0.6 $\mathrm{m} / \mathrm{s}$. It is shown when the flow field becomes stable with hydraulic mixing, stratification is obvious above the outlets (1.1 m-height). In the supernatant layer, the volume fraction of straw viscous fluid is very small, while that of water is nearly $100 \%$, a big quantity of straw settles at the bottom. It is as true as that the supernatant layer floats on the slurry layer. Though the stratification is not good for the mixing of fermentation slurry, the fermentation slurry is distributed relatively even below the supernatant layer, leaving a vertical channel with a small diameter in the inlet flow direction. In conclusion, the pressure outlets should be designed as high as possible in order to minimize the volume of supernatant layer, and to increase the effective fermentation volume.

\subsubsection{Inlet Velocity of $1 \mathrm{~m} / \mathrm{s}$}

Fig. 9 is the volume fraction contours of straw with the inlet velocity of $1 \mathrm{~m} / \mathrm{s}$. From the figure, it is found that the volume fraction distribution of straw in most area remains relatively even; the stratification in Fig. 5 


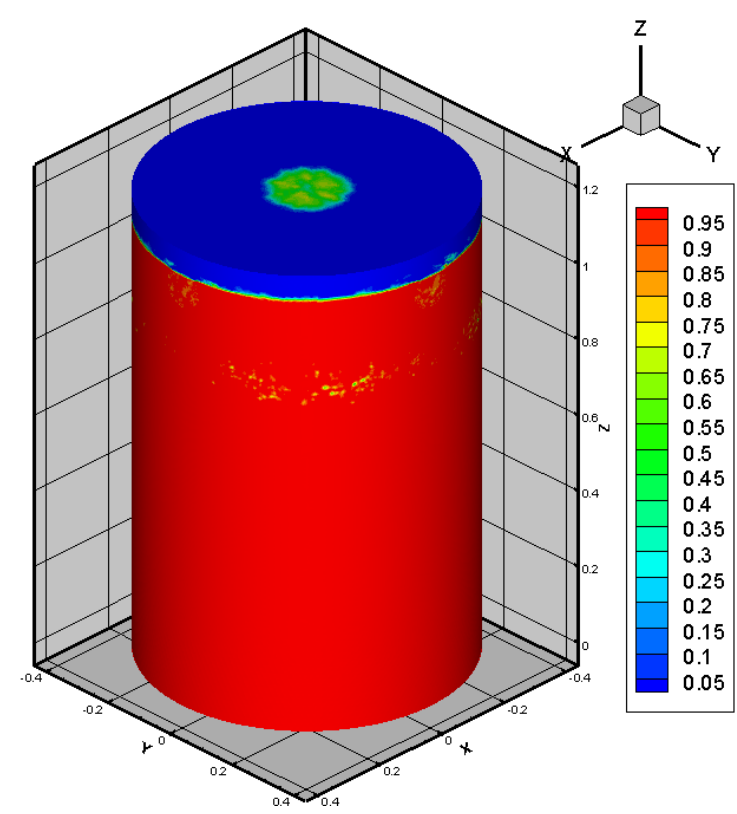

(a) $3 \mathrm{D}$

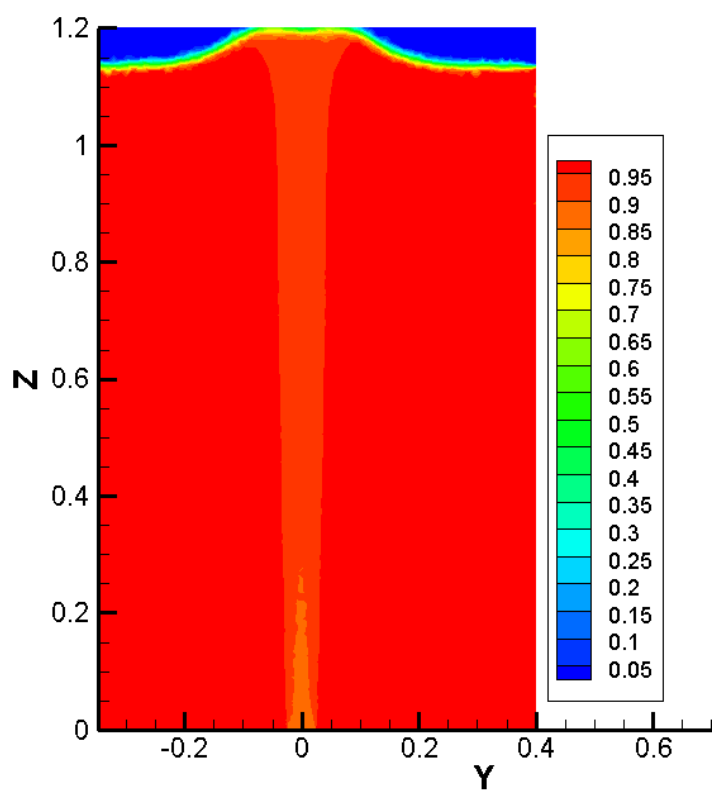

(b) $x=0$ section

Fig. 8 Inlet velocity $=0.6 \mathrm{~m} / \mathrm{s}$, volume fraction contours of phase 2 (straw).

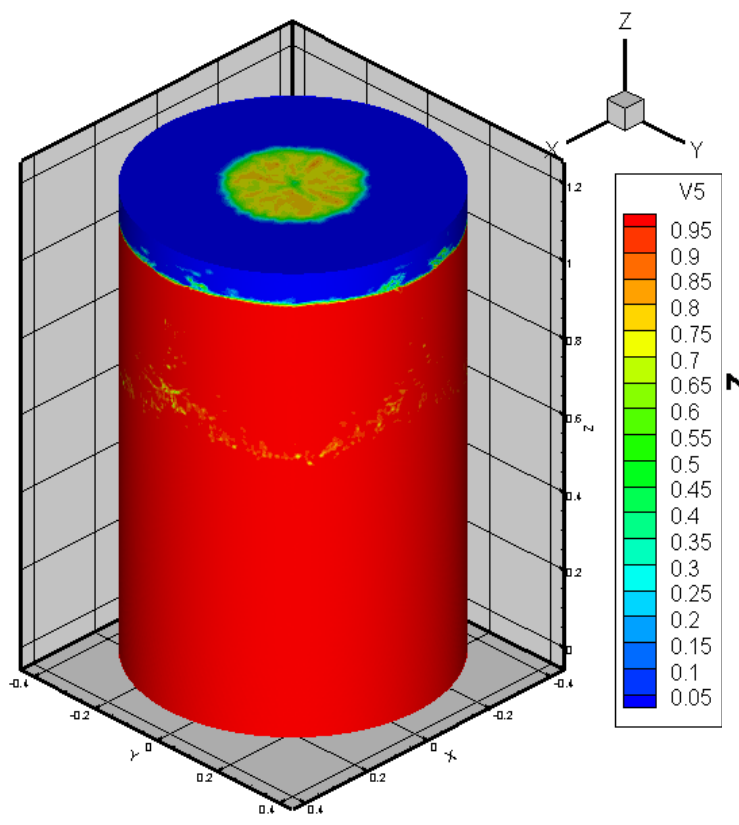

(a) $3 \mathrm{D}$

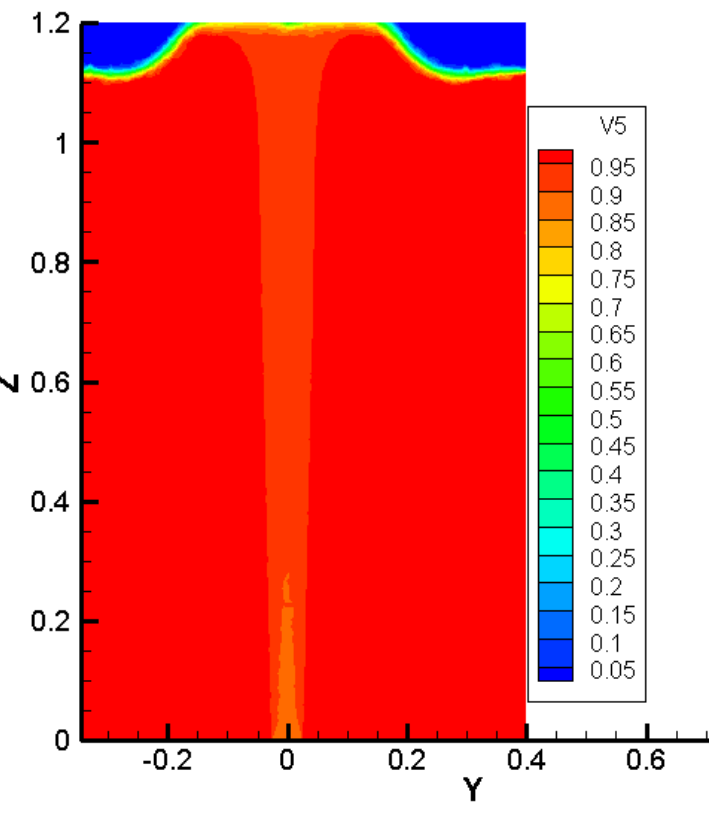

(b) $x=0$ section

Fig. 9 Inlet velocity $=1 \mathrm{~m} / \mathrm{s}$, volume fraction contours of straw.

does not occur in this working condition. At the same time, supernatant layer is compressed to be smaller due to the impact of a larger inlet velocity. Consecquently, the optimal inlet velocity is increased with the viscosity increase; therefore, inlet velocity needs to be promoted for a smaller volume of the supernatant layer.

\subsubsection{Inlet Velocity of $1.5 \mathrm{~m} / \mathrm{s}$}

Fig. 10 is the volume fraction contours of straw with the inlet velocity of $1.5 \mathrm{~m} / \mathrm{s}$. The figure indicates that the volume fraction distribution of straw is not quite uniform. Although the volume of supernatant layer drops, it is not completely broken, while another stratification occurs at the height of $z=0.25 \mathrm{~m}$, which 


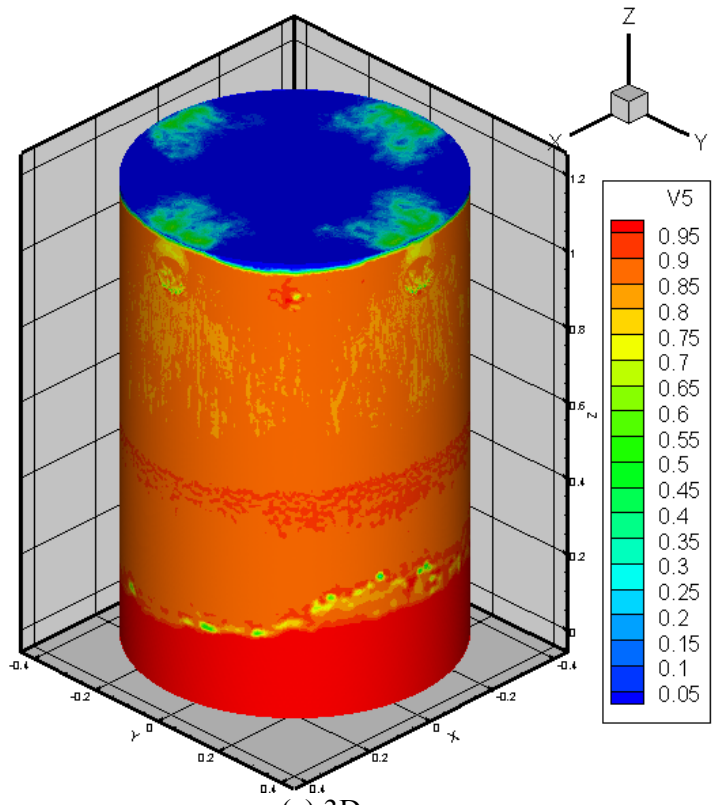

(a) $3 \mathrm{D}$

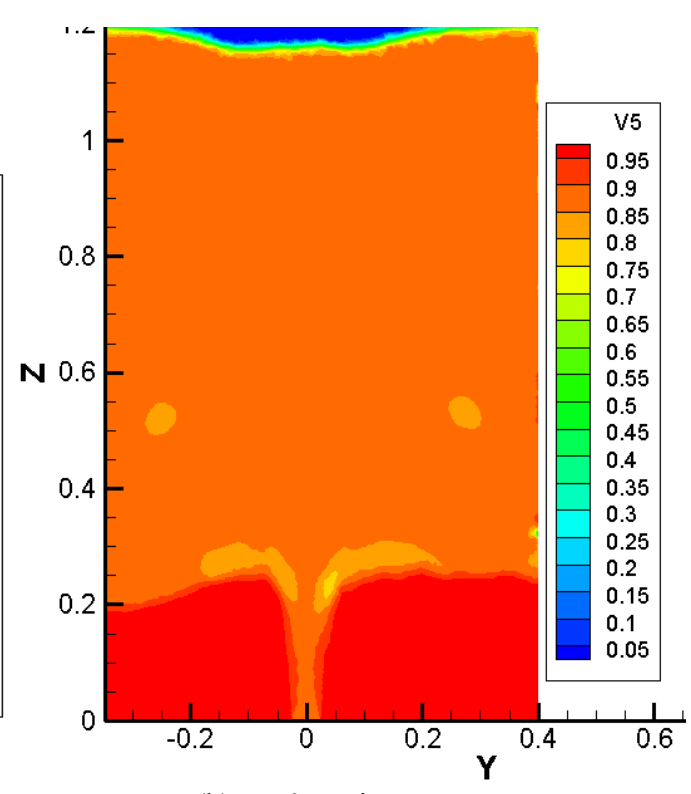

(b) $x=0$ section

Fig. 10 Inlet velocity $=1.5 \mathrm{~m} / \mathrm{s}$, volume fraction contours of straw.

divides the fluid region into two areas with sharply different concentrations. This may be caused by excess kinetic energy, which forms the vortex in the middle and lower positions. It can be seen that the excessive inlet velocity which may cause another stratification is against mixing, greatly reducing the mixture uniformity, wasting the effective volume, and against increasing gas production rate.

\section{Conclusions}

Using water as the medium, the basic flow field of an anaerobic digester can be simulated, and the optimal inlet velocity can be anticipated to match the digester structure and the mixing of the slurry for a higher gas production rate. The stratification of the supernatant layer and the fermentation layer cannot be avoided, but the volume of the supernatant layer can be relatively reduced if dispersed outlets are designed on high position and the inlet velocity is increased at the bottom. However, if the inlet velocity is too high, stratification will occur in the middle of the tank, leading to inconsistent mixing. In the case study presented in this paper, the basic flow field performs better when the inlet velocity is $0.6 \mathrm{~m} / \mathrm{s}$ rather than $1 \mathrm{~m} / \mathrm{s}$; however, during the later stage of fermentation, the viscosity increase causes the increase of optimal inlet velocity. No obvious stratification occurs when the inlet velocity is $1 \mathrm{~m} / \mathrm{s}$, more suitable than $0.6 \mathrm{~m} / \mathrm{s}$. While $1.5 \mathrm{~m} / \mathrm{s}$ is too large, and the excess kinetic energy will cause stratification towards the bottom area of the tank. In conclusion, in a particular anaerobic digester, the optimal inlet velocity increases with the viscosity increase; this can also be calculated quantitatively. In future work, a comparison experiment will be conducted using a number of anaerobic digesters with a bottom inlet and dispersed outlets on high position, where multiple feedstock is mixed at different inlet velocity to test the biogas producing rate and pollutants removal rate, and to verify the accuracy rate of CFD simulation.

\section{Acknowledgements}

The authors are indebted to the Ministry of Science and Technology of the People's Republic of China (MOST)-Sino-German Cooperation Program (No: 2011DFA62850) and the Sichuan Province Science and Technology Support Project (No: 2013GZ0107) for providing financial assistance. 


\section{References}

[1] Z.M. Lai, E.S. Long, Z.L. Mei, J. Du, T. Luo, R.Y. Huang, Utilization of multiphase flow technology for biogas industry integration, in: Low Carbon Town and Physical Energy Storage Proceedings, Changsha, China, 2013, pp. 245-249.

[2] L. Qiu, L.Y. Wang, P. Yang, Control on the dynamic fermentation technology of rural biomass project, Renewable Energy Resources 1 (2005) 53-55.

[3] H. Yang, L.W. Deng, G.Z. Zhang, Y.H. Lei, G.Z. Shi, A review on effects of stirring on biogas production of anaerobic digestion, China Biogas 28 (4) (2010) 3-10.

[4] X.J. Cai, L.Z. Wu, Explore the structure of the lateral inserted agitator, Petro-Chemical Equipment 30 (5) (2001) 45-46.

[5] Z.P. Chen, X.W. Zhang, X.H. Lin, The Stirring and Mixing Equipment Design Selection Manual, Chemical Industry Press, Beijing, China, 2004.

[6] Y. Liu, L.W. Deng, Z.Y. Wang, Rheological properties of several kinds of feedstocks for anaerobic fermentation and their influencing factors, Transactions of the CSAE 25 (8) (2009) 204-209.

[7] M.S. Vesvikar, M. Al-Dahhan, Flow pattern visualization in a mimic anaerobic digester using CFD, Biotechnology and Bioengineering 89 (2005) 732-739.

[8] K. Karim, R. Varma, M. Vesvikar, M.H. Al-Dahhan, Flow pattern visualization of a simulated digester, Water Research 38 (2004) 3659-3670.

[9] M.S. Vesvikar, R. Varma, K. Karim, M. Al-Dahhan, Flow pattern visualization in a mimic anaerobic digester experimental and computational studies, Water Science \& Technology 52 (2005) 537-543.

[10] B. Wu, S. Chen, CFD simulation of non-Newtonian fluid flow in anaerobic digesters, Biotechnology and Bioengineering 99 (3) (2008) 700-711.
[11] F. Shen, H. Yuan, Y. Pang, S Chen, B. Zhu, D. Zou, et al., Performances of anaerobic co-digestion of FVW (fruit \& vegetable waste) and FW (food waste): Single-phase vs. two-phase, Bioresource Technology 144 (2013) 80-85.

[12] B. Wu, CFD simulation of gas and non-Newtonian fluid two-phase flow in anaerobic digesters, Water Research 44 (2010) 3861-3874.

[13] R. Michelan, T.R. Zimmer, J.A.D. Ro-drigues, S.M.R.I D. deMaraes, M. Zaiat, E. Forest I, Effect of impeller type and mechanical mixing on the mass transfer and power consumption aspects of ASBR operation treating synthetic wastewater, Journal of Environmental Management 90 (2009) 1357-1364.

[14] A.G. Brito, A.C. Rodrigues, L.F Melo, Feasibility of a pulsed sequencing batch reactorwith an anaerobic aggre-gated biomass for the treatment of low strength wastewaters, Water Science and Technology 35 (1997) 193-198.

[15] S.R. Lee, N.K. Cho, W.J. Maeng, Using the pressure of biogas created during anaerobic digestion as the source of mixing power, Journal of Fermentation and Bioengineering 80 (4) (1995) 415-417.

[16] K. Karim, R. Hoffmann, T. Klasson, M.H.Al. Dahhan, Anaerobic digestion of animal waste: Waste strength versus impact of mixing, Bioresource Technology 96 (2005) 1771-1781.

[17] R.Y. Huang, J. Wang, T. Luo, E.S. Long, Z.L. Mei, Effects of multiphase flow water configuration mode on the flow optimization of biogas engineering fermentation pool, in: Low Carbon Town and Physical Energy Storage Proceedings, Hong Kong, China, 2013, pp. 65-69.

[18] Y.R. Chen, A.G. Hashimoto, Rheological properties of aerated poultry waste slurries, Transactions of the ASAE 19 (1) (1976) 128-133. 